\title{
Determinantes dos Investimentos Diretos Externos em Países em Desenvolvimento
}

Marcelo José Braga Nonnenberg IPEA

Mário Jorge Cardoso de Mendonça IPEA

\begin{abstract}
RESUMO
Basicamente, os determinantes dos investimentos diretos externos (IDE) podem ser relativos às firmas e a características dos países de origem - push factors - ou a fatores locacionais - pull factors. A maior parte da literatura teórica relativa a empresas multinacionais nos últimos quarenta anos, justamente por focar sua atenção nas empresas, enfatizou os fatores relacionados a vantagens das firmas, dando pouca importância aos fatores macroeconômicos. O objetivo desse trabalho é estimar, com base em dados em painel para 33 países para o período 1975-2000, os principais determinantes dos IDEs em direção aos países em desenvolvimento. Fatores como o tamanho e o ritmo de crescimento do produto, a qualificação da mão de obra, a receptividade em relação ao capital externo, o risco do país e o desempenho das bolsas de valores estão entre os principais determinantes de IDE. Além disso, por meio da aplicação de uma teste de causalidade no contexto de dados em painel, foi possível mostrar que o investimento direto externo não tem efeito positivo sobre o PIB. Ao contrário, esse procedimento lidade mostrou que o PIB do país é que tem efeito sobre a entrada de IDE.
\end{abstract}

\section{PALAVRAS-CHAVE}

investimento direto externo, empresas multinacionais, dados em painel

ABSTRACT

Basically, foreign direct investments (FDI) determinants may be referred to firms and country characteristics - push factors - or to locational factors - pull factors. Most of theoretical literature relative to multinational enterprises in the last forty years, has emphasized firms advantages, giving no weight to macroeconomic factors. The purpose of this paper is to estimate, based on panel data of 33 countries for the period 1975-2000, the main determinants of FDI inflows in developing economies. Factors as size and pace of growth of economic activity, the level of labor qualification, friendly policies towards foreign capital, country risk and stock market performance are the main determinants of FDI. In addition, a causality test, in the special context of panel data, has shown that FDI does not cause economic growth. On the contrary, it has been verified that economic growth causes FDI.

KEY WORDS

foreign direct investment, multinational enterprises, panel data

JEL Classification

F2I, F23

EST. ECON., SÃO PAULO, V. 35, N. 4, P. 631-655, OUTUBRO-DEZEMBRO 2005 


\section{INTRODUÇÃO}

A participação dos países em desenvolvimento no total de fluxos de ingresso de Investimentos Diretos Externos (IDEs) vem oscilando consideravelmente nos últimos 25 anos, passando de 15\% em 1980 para 46\% em 1982, e situando-se pouco acima dos $20 \%$ nos últimos quatro anos. Mais importante do que isso, entretanto, é perceber que as motivações para esses movimentos internacionais de capital ainda são substancialmente diferentes daquelas relacionadas aos fluxos de IDEs em direção aos países desenvolvidos, ainda que tenham se alterado nas últimas décadas. Hoje, por exemplo, a busca de recursos naturais, agrícolas ou minerais tem uma importância bem menor do que no início do século XX.

Por outro lado, o movimento contemporâneo desses fluxos é extremamente complexo e obedece a uma grande variedade de fatores ligados ao ambiente competitivo onde as firmas operam, às suas características e a fatores econômicos dos países de origem e dos hospedeiros.

O objetivo desse artigo é estimar, com base em dados de painel, os principais determinantes dos IDEs em direção aos países em desenvolvimento. Como será visto, fatores como o tamanho e o ritmo de crescimento do produto, a qualificação da mão-de-obra, a receptividade em relação ao capital externo, o risco do país e o desempenho das bolsas de valores estão entre os que exercem efeito sobre o IDE. Além disso, a partir da aplicação do teste de causalidade, no sentido Granger, no contexto de dados em painel, verificou-se causalidade simultânea entre IDE e PIB e não apenas num sentido específico, ou seja, o investimento direto externo sobre PIB, ou no sentido reverso.

Este estudo está estruturado da seguinte forma. A seção a seguir faz uma resenha da literatura teórica relativa aos determinantes do investimento direto externo. Na seção 2 são examinados trabalhos recentes que têm como enfoque a relação entre IDE e diversos fatores econômicos. A seção 3 apresenta o modelo e as hipóteses a serem testadas. A análise dos resultados é realizada na seção 4 . A seção 5 testa a relação de causalidade entre investimento externo e o produto. Por fim, a última seção apresenta as principais conclusões do trabalho.

\section{DETERMINANTES DOS IDEs: RESENHA TEÓRICA}

O objetivo desta seção é promover uma breve revisão das principais correntes da literatura teórica referente aos determinantes dos IDE. Uma resenha mais ampla pode ser vista em Nonnenberg (2002). Uma conclusão que sobressai é que a maior parte desses trabalhos dá mais ênfase a fatores específicos à firma multinacional, em especial os as- 
pectos ligados ao tipo de concorrência entre elas e as firmas locais, preocupando-se menos com os fatores locacionais.

Apesar de os primeiros trabalhos teóricos que se ocuparam dos determinantes dos IDEs remontarem a Adam Smith, Stuart Mill e Torrens, um dos primeiros teóricos a desenvolver argumentos a esse respeito foi Ohlin (1933). Segundo ele, os investimentos diretos externos eram motivados principalmente pela possibilidade de obtenção de altas taxas de lucro em mercados em crescimento, facilitados pela possibilidade de financiá-los a taxas de juros relativamente reduzidas no país de origem. Mas também constituíam fator determinante a necessidade de transpor barreiras comerciais e garantir fontes de matérias-primas.

Hymer (1960) inaugura uma nova tradição nos estudos sobre empresas multinacionais (EMN). Se as EMNs conseguem competir com empresas locais, que têm maior conhecimento do mercado e do ambiente local, é porque apresentam alguma vantagem compensatória. Essas podem ser:

- concorrência imperfeita, por exemplo, dada por diferenciação de produto;

- concorrência imperfeita no mercado de fatores, por exemplo, acesso a conhecimento patenteado ou próprio, discriminação no acesso a capital ou a diferenças de capacitação;

- economias de escala internas ou externas, inclusive as dadas por integração vertical;

- intervenção governamental, como restrições às importações.

Ocorrendo essas vantagens, a EMN irá preferir atender ao mercado externo por meio de investimentos diretos ao invés de exportações. Analogamente, não irá licenciar a produção para empresas locais quando estas estiverem incertas com relação ao valor da licença ou quando os custos de transferência do conhecimento para o detentor (direitos de propriedade) forem elevados.

Kindleberger (1969) modifica ligeiramente a análise de Hymer. Ao invés de a conduta da EMN determinar a estrutura dos mercados, é a estrutura - concorrência monopolística - que vai determinar a conduta da empresa que irá internacionalizar sua produção. Caves (1971) também desenvolve argumento semelhante, em que a estrutura ditará a conduta. Os IDEs vão ocorrer basicamente em setores dominados por oligopólios. Quando houver diferenciação de produtos, poderão ocorrer investimentos horizontais, isto é, no mesmo setor. Quando não houver diferenciação de produtos, haverá investimentos verticais, isto é, em setores para trás na cadeia produtiva da empresa. A existên- 
cia de IDE ainda se relaciona com barreiras ao comércio ou então para evitar incertezas de fornecimento ou como forma de levantar barreiras a novas firmas no mercado externo.

Assim, a hipótese de investimentos diretos determinados por ativos específicos que compensem a desvantagem inicial das empresas estrangeiras ante as firmas locais passou a constituir, na literatura, a tradição HKC, em homenagem a Hymer, Kindleberger e Caves. Markusen e Venables (1995) elaboraram modelo na mesma linha, comparando a importância das multinacionais com o comércio exterior. A presença das multinacionais (ante o comércio) aumenta à medida que os países ficam mais parecidos em termos de renda, dotação relativa de fatores e tecnologia.

Uma segunda linha de estudos sobre os determinantes dos IDEs baseia-se na idéia de internalização de custos de transação. Buckley e Casson (1976 e 1981) e Buckley (1985) foram os primeiros a explorar essa hipótese, partindo da idéia de que os mercados de produtos intermediários são imperfeitos, apresentando maiores custos de transação, quando a administração é feita por empresas diferentes. A integração dos mercados pela EMN, portanto, iria minimizar esses custos. As EMN possuem direitos de propriedade (proprietary assets) relativos a marketing, design, patente, marca, capacidade de inovação, etc., cuja transferência deve ter um custo elevado, por serem ativos intangíveis, ou em razão de oportunismo ou mesmo em razão de serem difusos e, portanto, de difícil venda ou aluguel.

A ênfase da teoria da internalização é nos mercados de produtos intermediários e na formação de redes internacionais de produção. Talvez sua principal força resida na capacidade de responder ao dilema entre licenciamento da produção por um agente no exterior ou produção própria. Assim, a escolha da firma é dupla: localização e modo de controle. Quando a produção e o controle são no país de origem, a firma exporta; quando a decisão é produção no exterior e controle local, ela licencia; e quando produz e controla no exterior, ocorre o IDE. Normalmente, essas escolhas correspondem a diversos estágios na internacionalização da produção.

Ainda dentro de uma visão que pode ser classificada como microeconômica estão os trabalhos de John Dunning. Sua análise começa pela constatação de que a propriedade de ativos diferenciados pode ser compreendida como um dos fatores responsáveis pela existência das multinacionais. Dunning vai desenvolver uma abordagem que, segundo ele, deve ser entendida como um paradigma (na medida em que pode englobar diversas teorias concorrentes, sem apresentar uma previsão única), conhecida pelas letras OLI (Ownership, Location, Internalization). Esse paradigma pode ser apresentado esquematicamente da seguinte forma: ${ }^{1}$

1 Dunning (1993, p. 79 e 80) 
A firma estrangeira apresenta vantagens sobre as firmas domésticas num determinado setor em decorrência da propriedade privilegiada (Ownership) de alguns ativos tangíveis ou intangíveis que estejam disponíveis apenas para as firmas daquela nacionalidade. (1)

Dado (1), a firma estrangeira irá usar sua vantagem de propriedade se achar mais vantajoso internalizá-la do que vendê-la, isto é, se possuir vantagem de internalização (I). (2)

Dados (1) e (2), a firma estrangeira irá produzir no país de destino se aí existirem as vantagens locacionais (L) suficientes para justificar a produção nesse país e não em qualquer outro. Dunning ainda acrescenta uma quarta condição, que é a compatibilidade do investimento externo com a estratégia de longo prazo da multinacional.

Com base nesse paradigma, Dunning apresenta quatro razóes para que a firma invista no exterior: busca de recursos, busca de mercados, busca de eficiência e busca de ativos estratégicos A articulação entre os ativos de conhecimento e os fatores locacionais está por trás da motivação de investimentos das EMNs, que experimentou o mais rápido crescimento nas últimas décadas, qual seja, a busca de ativos estratégicos. Não obstante ter havido um grande crescimento, nos últimos anos, do deslocamento das EMNs em direção a países em desenvolvimento, em busca de mercados ou em busca de recursos (market-seeking ou resource-seeking), a principal novidade é o crescimento dos investimentos nos países desenvolvidos em busca de ativos estratégicos, mudando a natureza do fator locacional. Se, por um lado, ativos de conhecimento (intangíveis) são móveis entre fronteiras e, portanto, as empresas multinacionais obtêm vantagens competitivas ao buscar ativos estratégicos em países distintos da sua origem, por outro, a localização daqueles ativos é cada vez mais influenciada pela existência de clusters de atividades complementares entre si.

Uma outra corrente de estudos é representada pelo modelo de ciclo de produto de Vernon (1966). De acordo com esse modelo, como as inovações são poupadoras de mãode-obra, surgem inicialmente nos países mais intensivos em capital, em especial nos EUA (o modelo foi elaborado em meados da década de 60). Paulatinamente, a produção vai sendo deslocada para países menos intensivos em capital e, finalmente, para países em desenvolvimento. Ao mesmo tempo, a produção nos países mais ricos vai sendo reorientada para novos produtos ligados às últimas inovaçôes de produtos e processos. Esse modelo foi parcialmente responsável por um conjunto de estudos ${ }^{2}$ sobre o processo de disseminação das empresas multinacionais concebido como sendo seqüencial em estágios. Assim, as empresas iriam, inicialmente, atender aos mercados pela exportação para, posteriormente, estabelecer representantes comerciais e, finalmente, começar a produzir nos mercados de destino por meio de subsidiárias.

2 Quase todos encontram-se em Buckley e Ghauri (1991). 
Ainda na linha de internacionalização da produção por razões endógenas à firma, encontram-se os trabalhos de Graham (1978, 1998 e 2000). Neles, o aparecimento de EMN resulta da interação oligopolística à medida que as firmas crescem, como estratégia de redução de riscos. No trabalho mais recente, o autor usa a teoria dos jogos para desenvolver um modelo simplificado com dois países e um setor para explorar a entrada de uma firma num país estrangeiro como reação à entrada de firma de outro país no seu mercado local. O ponto relevante a enfatizar é que a rivalidade entre firmas é uma dimensão importante e nem sempre levada em conta nas explicações sobre as EMNs.

Uma das correntes recentes mais promissoras é a que segue os trabalhos de Cantwell (resumidos em Cantwell, 2000). Parte do conceito de acumulação tecnológica como um processo interno à firma e cumulativo. Isto é, como a tecnologia é tácita, não é transferida sem custos, cada firma irá desenvolver suas capacitaçôes e seu conhecimento de forma única e diferenciada. A competição entre firmas é, basicamente, tecnológica. É a inovação que é o principal gerador de lucros. A internacionalização da produção permite à firma utilizar seu conhecimento e potencial inovador em outros ambientes, ampliando suas vantagens específicas. Além disso, a expansão leva à adaptação, que permite mais inovação.

Por essa razão, a firma irá desenvolver seu conhecimento em redes internacionais de tecnologia, realizando investimentos em outros centros de forma a ter acesso a novos conhecimentos. Ao contrário do paradigma Hymer-Kindleberger-Caves, a internacionalização da produção é resultado da maior concorrência tecnológica.

\section{DETERMINANTES DOS IDEs: TRABALHOS EMPÍRICOS}

Os trabalhos empíricos que procuram estimar a importância dos diversos determinantes dos IDEs enfatizam mais os fatores de atração, ou seja, locacionais, até porque os dados raramente permitem identificar os países de origem dos investimentos, ao menos para um grande conjunto de países e anos. As vantagens de propriedade do capital, por exemplo, apontadas por alguns trabalhos na seção l como um importante push-factor, são de mais difícil observação, dependendo de pesquisas envolvendo firmas. As principais variáveis utilizadas são, normalmente, o tamanho do mercado, a taxa de crescimento do PIB, a estabilidade econômica, o grau de abertura da economia, além de diversas variáveis institucionais, como será visto adiante.

Entretanto, a relação entre IDE e crescimento econômico merece uma atenção especial. Se, por um lado, o crescimento econômico pode ser um poderoso estímulo ao ingresso de IDE, por outro, o aumento dos investimentos externos - na medida em que constituem elevação do estoque de capital existente (greenfield investment) - também pode 
ser apontado como um dos fatores responsáveis pelo crescimento econômico, significando a existência de um problema de endogeneidade. Há, assim, outros estudos que se ocupam em provar a relação entre IDE e nível de atividade.

Com relação aos determinantes dos IDEs, é preciso notar que há diferenças substanciais entre os fluxos que envolvem apenas países desenvolvidos, tanto entre os países de origem como os hospedeiros e aqueles em que os hospedeiros são países em desenvolvimento. De acordo com Dunning (2002), no primeiro caso ocorrem investimentos do tipo procura de ativos estratégicos, em que o IDE é representado por uma operação de fusão e aquisição, e do tipo procura de eficiência horizontal. Já no segundo caso, os investimentos são caracterizados por procura de mercados, de recursos ou de eficiência horizontal.

Os trabalhos examinados a seguir referem-se, assim, a determinantes de IDE dirigidos a países em desenvolvimento. Nunnenkamp e Spatz (2002), usando amostra de 28 países em desenvolvimento para o período 1987-2000, encontram correlaçóes de Spearman significativas entre fluxos de IDE e PIB per capita, fatores de risco, anos de escolaridade, restrições ao comércio externo, fatores complementares de produção, ${ }^{3}$ gargalos administrativos ${ }^{4}$ e fatores de custo. ${ }^{5}$ População, crescimento do PIB, restriçõoes à entrada de firmas, restrições após a entrada e regulação relacionada à tecnologia revelaram-se não significativas. No entanto, quando são feitas regressões, separadamente para os fatores não tradicionais, usando como controles os fatores tradicionais (população e PIB per capita), apenas custo de fatores apresenta resultados significativos, ainda assim apenas para 1997-2000.

Holland e outros (2000) fazem resenha de diversos trabalhos relacionados à Europa Oriental e Central, que mostram evidências da importância do tamanho do mercado e do potencial de crescimento como determinantes dos IDEs. Tsai (1994) analisa as décadas de 1970 e 1980 e enfrenta o problema da endogeneidade entre IDE e crescimento, desenvolvendo um sistema de duas equações simultâneas. Além disso, o IDE é medido alternativamente como fluxo e estoque. O tamanho do mercado resulta mais importante para o fluxo de IDE do que o crescimento. O superávit comercial apresenta sinal negativo e significativo para o IDE, ao passo que o fluxo de IDE diminui à medida que o salário nominal diminui. Por outro lado, o impacto do IDE sobre crescimento econômico é bastante limitado.

3 Insumos locais necessários à produção competitiva internacionalmente, com base em pesquisa conduzida por European Round Table of Industrialists (2000) - ERT.

4 Também ERT (2000).

5 Relacionados a impostos, condições de emprego, regulação do mercado de trabalho e poder de sindicatos, também de acordo com ERT (2000). 
Campos e Kinoshita (2003) utilizam dados em painel para analisar 25 economias em transição entre 1990 e 1998. Conclui-se que, para esse conjunto de países, o IDE é influenciado sobretudo por economias de aglomeração (clusters), tamanho do mercado, baixo custo da mão-de-obra e recursos naturais abundantes. Além desses fatores, as seguintes variáveis apresentam resultados significativos: boas instituiçôes, abertura comercial e menores restrições aos fluxos de IDE.

Garibaldi e outros (2001), com base em painel dinâmico para 26 países em transição entre 1990 e 1999, analisam um grande conjunto de variáveis, divididas em fundamentos macroeconômicos, reformas estruturais, quadro legal e institucional, condições iniciais e percepção de risco. Os resultados indicam que as variáveis macroeconômicas, como tamanho do mercado, déficit fiscal, inflação e regime cambial, de percepção de risco, reformas econômicas, liberalização comercial, dotação de recursos naturais, barreiras ao investimento e burocracia têm o sinal esperado e são significativas.

Loree e Guisinger (1995), estudando os determinantes dos investimentos diretos externos dos Estados Unidos em 1977 e 1982 (tanto em países desenvolvidos quanto em países em desenvolvimento), concluem que variáveis relacionadas a políticas nos países hospedeiros são significativas em países desenvolvidos apenas enquanto a infra-estrutura é um determinante importante em todas as regiões.

Os trabalhos a seguir procuram ou determinar a influência dos IDEs sobre a taxa de crescimento do PIB ou analisar a causalidade reversa entre essas duas variáveis. Borensztein e outros (1995) utilizam dados para o período 1970 a 1989 envolvendo fluxos dos países desenvolvidos para os em desenvolvimento. As principais conclusões são que, em primeiro lugar, o IDE tem efeito positivo sobre o crescimento econômico, dependendo do estoque de capital humano disponível na economia hospedeira. Entretanto, onde o nível de capital humano é muito baixo, o efeito é negativo. Em segundo lugar, possui um efeito indireto sobre o crescimento na medida em que atrai atividades complementares.

Mello (1999) considera que o IDE afeta o crescimento por meio da acumulação de capital e também via transferência de conhecimento. Essas hipóteses são testadas com base em análise de séries temporais e de dados em painel. Os resultados de séries temporais não são conclusivos. Os dados em painel mostram que o IDE afeta positivamente o crescimento por meio da transferência de conhecimento nos países da OECD, mas não nos demais. Já o efeito sobre acumulação de capital manifesta-se apenas nos países não-OECD. Esses resultados indicam que o efeito final depende da complementaridade ou da substituição entre investimentos externos e domésticos. 
Os diversos resultados obtidos por Lipsey (2000) permitem inferir que o efeito do IDE sobre o crescimento é positivo, porém reduzido, e depende fortemente da interação com o nível de escolaridade do país receptor. Soto (2000), trabalhando com dados em painel para países em desenvolvimento para o período 1986-97, conclui que o IDE contribui positivamente para o crescimento por meio da acumulação de capital e da transferência de tecnologia.

Um teste de causalidade entre IDE e crescimento do produto é proposto por Nair-Reichert e Weinhold (2001), com base em dados em painel para 24 países em desenvolvimento para o período 1971-85. A principal conclusão é que a relação entre investimentos, tanto externos quanto domésticos, e crescimento do produto é fortemente heterogênea e que a eficácia do IDE é positivamente influenciada pelo grau de abertura do país.

Finalmente, Buckley e outros (2002) utilizam dados em painel para diversas regióes da China no período 1989-98. Primeiramente, é visto que se a taxa de crescimento de IDE afeta positivamente o crescimento do PIB, o reverso não é verdadeiro. Segundo, não são encontradas evidências que apóiem a tese segundo a qual a eficácia do IDE depende de um patamar mínimo de capital humano. Em contraste, o capital humano é mais significativo nas províncias menos desenvolvidas, ao passo que o IDE favorece o crescimento especialmente nas províncias mais desenvolvidas.

\section{MODELO ECONOMÉTRICO}

A seção anterior mostrou que faltam estudos compreendendo uma amostra representativa de países em desenvolvimento que utilize a metodologia de dados em painel para analisar os determinantes dos IDE. E, como será evidenciado a seguir, essa metodologia pode apresentar resultados superiores, permitindo conclusões mais precisas.

O objetivo desta seção é apresentar o modelo que será utilizado para testar empiricamente o grau de influência das variáveis mencionadas anteriormente sobre o investimento direto. A metodologia empregada é de dados em painel, que combina informação relativa à variação ao longo das unidades individuais, no caso os países em desenvolvimento, com informação que ocorre no tempo. A vantagem do emprego de dados em painel é que esse método permite levar em consideração as características idiossincráticas (heterogeneidade) existentes entre os grupos (países).

A seleção das variáveis representativas dos determinantes dos IDEs foi necessariamente afetada pela opção de se utilizar uma amostra suficientemente ampla de países em desenvolvimento como hospedeiros. A amostra compreende um conjunto de 33 países, 
sendo que o período se estende de 1975 a 2000. Assim, diversas variáveis importantes do ponto de vista teórico, e incluídas em alguns dos estudos analisados acima para um conjunto restrito de países, como aquelas relativas à estabilidade política, por exemplo, ficaram de fora. A lista dos países e a fonte dos dados são apresentadas no Apêndice.

As variáveis selecionadas para explicar o investimento direto nos países em desenvolvimento são o produto (PIB), taxa média de crescimento do PIB nos últimos cinco anos (G5PIB), a qualificação da força de trabalho (ESCOL), o grau de abertura comercial (ABERT), a taxa de inflação (INFLAÇÃO), a taxa de risco (RISCO), o consumo per capita de energia (ENERCON), o índice Dow Jones (DOWJONES) e o somatório do PIB dos maiores exportadores de capital da OECD (PIBOECD) para países em desenvolvimento.

Vamos, inicialmente, examinar as principais hipóteses sobre o comportamento esperado das variáveis explicativas do modelo. Com relação ao PIB, a intuição aqui está no fato de que o produto do país funciona como um fator de atração, uma vez que boa parte dos investimentos que se dirigem a países em desenvolvimento se encaixa no que Dunning (1993) denomina de investimentos market-seeking, como já foi colocado anteriormente. Assim, tal como em outros trabalhos, espera-se que a correlação encontrada seja positiva.

Conforme foi colocado, alguns estudos mostram existir correlação positiva entre os investimentos diretos e o crescimento do produto. A hipótese a ser testada neste trabalho é de que os IDEs reagem positivamente não ao crescimento corrente do produto, mas ao grau recente de estabilidade do crescimento apresentado num período de alguns anos. Uma suposição implícita aqui é que os agentes econômicos privilegiam mais a informação recente em detrimento daquela gerada num período de tempo distante. Para testar essa hipótese, iremos incluir como um dos determinantes do IDE a taxa média de crescimento do produto definida nos últimos cinco anos, representada aqui por G5PIB.

Há grande evidência empírica a respeito da relação positiva entre IDE e o nível de qualificação da mão-de-obra, aqui medida pelo porcentual da população em idade correspondente matriculada no ensino secundário - ESCOL. Também o nível de abertura comercial deve ser positivamente correlacionado com o investimento direto, pelo fato dessa variável representar uma boa proxy para o tipo de relação que um país tem com o capital externo. Supóe-se que um país com maior grau de abertura comercial e, portanto, com economia mais direcionada para fora, seja mais propenso a facilitar a entrada de investimento externo - ABERT. 
A taxa de inflação (INFLAÇÃO) serve como proxy para o grau de estabilidade econômica, tendo em vista que um dos sintomas clássicos da perda de controle, tanto do lado fiscal como monetário, é o descontrole inflacionário. Tomando por base que os investidores preferem países que apresentam economias mais estáveis, com menor grau de incerteza, é previsível que se observe um efeito negativo da inflação sobre o investimento direto.

O risco país, apesar de mais estreitamente ligado às condições de crédito, também deve influenciar o ingresso de IDE. Quanto maior o risco, maior a probabilidade de défault da dívida externa e, conseqüentemente, da adoção de medidas de restrição à saída de capitais internacionais. Dessa forma, o risco está negativamente associado ao ingresso de IDE. Aqui a variável RISCO será representada pela medida de risco conforme calculada pelo Euromoney.

A maior sofisticação da estrutura industrial de um país favorece os fluxos de IDE do tipo strategic asset seeking ou efficiency seeking, na classificação de Dunning. Para medir o grau de desenvolvimento da estrutura industrial foi introduzida a variável ENERCON, que mede o consumo per capita de energia nos países hospedeiros, com um sinal esperado positivo.

Sobretudo nos últimos anos, os IDEs estiveram muito associados a processos internacionais de fusões e aquisições que, por sua vez, foram fortemente influenciados pelo desempenho das bolsas. Assim, períodos que registram aumentos significativos neste índice - DOWJONES - refletem momentos de euforia com aumento da propensão a realizar investimentos com maior risco. Portanto, acreditamos que existe uma relação positiva entre os investimentos diretos nos países em desenvolvimento e a evolução dessa variável.

A variável GPIBOECD resulta da média da taxa de crescimento do produto dos maiores exportadores de IDE. ${ }^{6}$ A relação esperada entre esta variável e o IDE é, até certo ponto, ambígua. De um lado, uma correlação positiva entre PIBOECD e FDI pode ser explicada pelo aumento de maiores lucros e, portanto, mais recursos disponíveis para a realização de investimentos no exterior. De outro, uma correlação negativa entre essas duas variáveis pode ser interpretada como diversificação de risco.

A tarefa seguinte diz respeito à aplicação efetiva da metodologia de dados em painel para tratar o investimento direto. Em geral, uma regressão para dados em painel costuma aparecer da seguinte forma:

6 Ver Apêndice. 


$$
y_{i t}=\beta^{\prime} x_{i t}+v_{i t}, \quad i=1, \ldots, N ; t=1, \ldots, T
$$

$\operatorname{com} v_{i t}=\alpha_{i}+u_{i t}$

A variável dependente $y_{i t}$ é representada no modelo pelo investimento direto, enquanto $x_{i t}$ representa o vetor de variáveis explicativas que variam em i e t. Aqui $\alpha_{i}$ é um termo estocástico próprio das unidades, de forma que $\alpha_{i} \sim\left(0, \sigma_{\alpha}{ }^{2}\right)$, que se denomina efeito individual, enquanto que $u_{i t}$ é um distúrbio estocástico tal que $u_{i t} \sim\left(0, \sigma_{u}{ }^{2}\right)$. Temos ainda que $E\left[u_{i t} \alpha_{i}\right]=0$ e $E\left[u_{i t} x_{i t}\right]=0$. O efeito individual $\alpha_{i}$, próprio das unidades, pode ser ou não correlacionado com o vetor de variáveis explicativas $x_{i t}$. A existência de correlação entre o efeito individual e os regressores pode ser detectada por meio da aplicação do teste de Hausman (1978), cuja hipótese nula é de não correlação entre $\alpha_{i}$ e $x_{i t}$. No caso de haver correlação, a estimação deve ser feita a partir do estimador de efeito fixo (LSDV), ${ }^{7}$ caso contrário, o estimador de efeito aleatório é o mais apropriado.

No caso corrente temos que $x_{i t}=[\mathrm{PIB}$, ESCOL, G5PIB, ABERT, INFLAÇÃO, RIS$\mathrm{CO}$, ENERCON]. Se no modelo apresentado estivessem presentes somente variáveis do tipo $x_{i t}$, a estimação poderia se dar de modo convencional. Contudo, existem na nossa base de dados duas variáveis que somente variam no tempo e não nas unidades individuais, ou seja, países. Deste modo, para tratar este caso mais geral, o modelo da equação (1) deve ser reescrito do seguinte modo: (Hsiao, 2003)

$$
y_{i t}=\beta^{\prime} x_{i t}+\delta^{\prime} z_{t}+\alpha_{i}+\lambda_{t}+u_{i t}, \quad i=1, \ldots, N ; t=1, \ldots, T
$$

onde $z t$ é o vetor de variáveis que somente possuem variação em $t$. No nosso caso, temos que $z_{t}=$ [DOWJONES, GPIBOECD]. Notamos agora a presença do termo $\lambda_{t}$ especificamente relacionado à variação temporal, que compõe a classe dos distúrbios da regressão com $\lambda_{i} \sim\left(0, \sigma_{z}{ }^{2}\right), E\left(\lambda_{t} v_{i t}\right)=0$. Iremos ainda assumir que $z_{t}$ é não correlacionado com qualquer distúrbio.

Nosso objetivo, então, é obter uma estimativa fidedigna para o vetor de parâmetros $(\beta, \delta)$, o que será feita por meio da aplicação de um procedimento que aparece em Hsiao (2003) realizado em dois estágios. A solução pode ser se obtida estimando $\beta$ por efeito fixo restrito apenas às variáveis $x_{i t}$, para depois estimarmos $\delta$ a partir da aplicação do estimador OLS na seguinte regressão:

7 Least Square Dummy Variable. 


$$
\bar{y}_{t}-b_{W}{ }^{\prime} \bar{x}_{t}=u^{*}+\delta^{\prime} z_{t}+\lambda_{t}+\bar{u}_{t}, \quad t=1, \ldots, T
$$

onde $\bar{y}_{t}=\frac{1}{N} \sum_{i=1}^{N} y_{i t}, \quad \bar{x}_{t}=\frac{1}{N} \sum_{i=1}^{N} x_{i t}$, sendo $b_{w}$ o coeficiente estimado de $\beta$ obtido

por efeito fixo e $u^{*}$ o termo constante da equação. Deve-se ter em mente que para que o procedimento que aparece na equação (3) possa ser aplicado, é necessário que o teste de Hausman rejeite a hipótese nula de não correlação entre $\alpha_{i}$ e $x_{i t}$.

\section{ANÁLISE DOS RESULTADOS}

Na Tabela 1 são apresentados os resultados econométricos para o modelo de investimento estimado a partir de dados em painel, de acordo com o exposto acima. Os dados utilizados são anuais, abrangendo 33 países no período de 1975-2000. A razão para esse corte temporal é que, para os anos anteriores a 1975, algumas das variáveis utilizadas no modelo não estão disponíveis para boa parte dos países. Tendo em vista que a variável RISCO, importante para explicar o investimento direto, somente está disponível a partir de 1985, o modelo foi estimado para duas amostras: completa (1975-2000) e parcial (1985-2000). As variáveis PIB e PIBOECD foram tomadas em logaritmo.

Para cada uma dessas amostras, as estimativas foram geradas pelos seguintes modelos: (i) OLS (pooling ou dados agrupados), (ii) painel efeito aleatório, (iii) painel efeito fixo. Neste último os coeficientes das variáveis que possuem apenas variação temporal foram estimados com base na metodologia descrita pela equação (3). A análise aqui segue próxima àquela que aparece em Hausman e Taylor (1981) e Cornwell e Rupert (1988). A estimação por OLS de dados agrupados (pooling) aparece apenas como referência. Ela permite checar a existência de multicolinearidade no modelo por meio da estatística Variance Inflation Factor. A literatura assinala que existe indicação de multicolinearidade se o valor VIF exceder ao valor 5. (Judge et alii, 1982). A estimação por OLS pode dar ainda alguma idéia do ganho de eficiência em se estimar o modelo por dados em painel.

Conforme pode ser observado na Tabela 1, existe ganho de eficiência advindo do emprego de dados em painel. Em todos os modelos nos quais se faz uso deste método a estatística RHO e o teste de Breusch-Pagan revelam a importância do componente individual. A estatística RHO mostra que a proporção estimada da variância do componente individual em relação à variância total do distúrbio é alta. A necessidade de levar em conta as diferenças individuais ainda é corroborada pelo teste de Breusch-Pagan, cuja hipótese nula é que a variância do componente individual $\alpha_{i}$ seja igual a zero. Os 
resultados mostram, ainda, que existe uma clara preferência para a aplicação do estimador de efeito fixo, pois o teste de Hausman indica presença de correlação entre o componente individual e as variáveis explicativas. Assim, os modelos mais fidedignos para cada uma das amostras usadas são os que aparecem nas colunas (iii) e (vi).

Observa-se ainda que em ambos os modelos de dados agrupados (pooling) as variáveis ESCOL, INFLAÇÃO e G5PIB são não significativas. Também para o modelo de dados agrupados, estimado com base no período restrito 1985-2000, a variável RISCO não se mostrou significativa. Diferentemente, todas estas variáveis se mostram significativas nos modelos estimados a partir da metodologia de dados em painel. Além disso, todas as variáveis são significativas, apresentando os sinais esperados.

De acordo com a Tabela 1, os resultados encontrados para os modelos (iii) e (vi) corroboram muitas das hipóteses formuladas inicialmente. De fato, a taxa média de crescimento do produto num período anterior - G5PIB, nos dois modelos de efeito fixo, é significativa, com coeficiente positivo. A taxa corrente de crescimento do produto foi testada e não mostrou significância. Observou-se, tal como era esperado, que o log do produto (LPIB) também exerce influência positiva sobre o investimento direto. As variáveis ESCOL e ABERT apresentam coeficientes estimados positivos, além de serem significativas.

Tendo ainda em vista os resultados da Tabela 1 , a inflação tomada como proxy para estabilidade macroeconômica possui, conforme era esperado, correlação negativa com o investimento direto externo. Contudo, para o período mais restrito, essa variável se mostrou pouco significativa, dados os parâmetros usuais. É importante ressaltar que a variável RISCO é fortemente significativa, apresentando o sinal esperado. Isto mostra que essa variável é importante não apenas para explicar os fluxos financeiros, conforme a hipótese usual, mas também para os fluxos de IDE.

Por fim, no que se refere aos componentes exógenos do modelo, que somente possuem variação temporal, o índice DOW JONES apresenta significância, sendo positivamente correlacionado com IDE. Por outro lado, o consumo de energia não se mostrou significativo em nenhuma das duas amostras. GPIBOECD só é significativa (mesmo assim a 10\%) quando não se introduz RISCO. 


\section{TABELA 1 - MODELO DE DADOS EM PAINEL PARA O INVESTIMENTO EX- TERNO DIRETO}

\begin{tabular}{|c|c|c|c|c|c|c|}
\hline \multirow{3}{*}{$\begin{array}{l}\text { Variável Depen- } \\
\text { dente = } \\
\text { Variáveis } \\
\text { Independentes = }\end{array}$} & \multicolumn{6}{|c|}{ Log do Investimento Direto Externo (LIDE) } \\
\hline & \multicolumn{3}{|c|}{$1975-2000$} & \multicolumn{3}{|c|}{$1985-2000$} \\
\hline & $\begin{array}{c}\text { OLS } \\
\text { (Pooling) } \\
\text { (I) }\end{array}$ & $\begin{array}{c}\text { Efeito } \\
\text { Aleatório } \\
\text { (II) }\end{array}$ & $\begin{array}{l}\text { Efeito } \\
\text { Fixo } \\
\text { (III) }\end{array}$ & $\begin{array}{c}\text { OLS } \\
\text { (Pooling) } \\
\text { (IV) }\end{array}$ & $\begin{array}{c}\text { Efeito } \\
\text { Aleatório } \\
\text { (V) }\end{array}$ & $\begin{array}{l}\text { Efeito } \\
\text { Fixo } \\
(\mathrm{VI})\end{array}$ \\
\hline LPIB (it) & $\begin{array}{l}1.0010 \\
(0.000)\end{array}$ & $\begin{array}{l}1.3204 \\
(0.000)\end{array}$ & $\begin{array}{l}1.4834 \\
(0.000)\end{array}$ & $\begin{array}{l}1.0541 \\
(0.000)\end{array}$ & $\begin{array}{l}1.2677 \\
(0.000)\end{array}$ & $\begin{array}{l}1.7282 \\
(0.000)\end{array}$ \\
\hline G5PIB (it) & $\begin{array}{l}0.0145 \\
(0.221)\end{array}$ & $\begin{array}{l}0.0458 \\
(0.001)\end{array}$ & $\begin{array}{l}0.0481 \\
(0.000)\end{array}$ & $\begin{array}{l}0.0333 \\
(0.005)\end{array}$ & $\begin{array}{l}0.0553 \\
(0.000)\end{array}$ & $\begin{array}{l}0.0510 \\
(0.000)\end{array}$ \\
\hline ESCOL (it) & $\begin{array}{l}0.0006 \\
(0.818)\end{array}$ & $\begin{array}{l}0.0172 \\
(0.001)\end{array}$ & $\begin{array}{l}0.0266 \\
(0.000)\end{array}$ & $\begin{array}{r}-0.0015 \\
(0.601)\end{array}$ & $\begin{array}{l}0.0134 \\
(0.013)\end{array}$ & $\begin{array}{l}0.0250 \\
(0.000)\end{array}$ \\
\hline ABERT (it) & $\begin{array}{l}0.0113 \\
(0.000)\end{array}$ & $\begin{array}{l}0.0143 \\
(0.000)\end{array}$ & $\begin{array}{l}0.0160 \\
(0.000)\end{array}$ & $\begin{array}{l}0.0115 \\
(0.000)\end{array}$ & $\begin{array}{l}0.0127 \\
(0.000)\end{array}$ & $\begin{array}{l}0.0146 \\
(0.000)\end{array}$ \\
\hline INFLAÇÃO (it) & $\begin{array}{r}-0.0001 \\
(0.641)\end{array}$ & $\begin{array}{r}-0.0002 \\
(0.024)\end{array}$ & $\begin{array}{r}-0.0002 \\
(0.019)\end{array}$ & $\begin{array}{r}-0.0001 \\
(0.564)\end{array}$ & $\begin{array}{r}-0.0001 \\
(0.061)\end{array}$ & $\begin{array}{r}-0.0001 \\
(0.108)\end{array}$ \\
\hline RISCO (it) & - & - & - & $\begin{array}{r}-0.2055 \\
(0.971)\end{array}$ & $\begin{array}{r}-20.9666 \\
(0.001)\end{array}$ & $\begin{array}{r}-20.4480 \\
(0.001)\end{array}$ \\
\hline ENERCON (it) & $\begin{array}{r}-0.0003 \\
(0.000)\end{array}$ & $\begin{array}{r}-0.0001 \\
(0.365)\end{array}$ & $\begin{array}{r}-0.0001 \\
(0.713)\end{array}$ & $\begin{array}{r}-0.0003 \\
(0.000)\end{array}$ & $\begin{array}{r}-0.0001 \\
(0.000)\end{array}$ & $\begin{array}{r}-0.0001 \\
(0.316)\end{array}$ \\
\hline DOWJONES (t) & $\begin{array}{l}0,0003 \\
(0.000)\end{array}$ & & $\begin{array}{l}0.0002 \\
(0,000)\end{array}$ & $\begin{array}{l}0.0002 \\
(0.000)\end{array}$ & & $\begin{array}{l}0.0001 \\
(0.000)\end{array}$ \\
\hline GPIBOECD (t) & $\begin{array}{r}-0.0434 \\
(0,209)\end{array}$ & & $\begin{array}{r}-0.0649 \\
(0.097)\end{array}$ & $\begin{array}{r}-0.0118 \\
(0.817)\end{array}$ & & $\begin{array}{l}0.0088 \\
(0.849)\end{array}$ \\
\hline CONSTANTE & $\begin{array}{l}-5.888 \\
(0.000)\end{array}$ & $\begin{array}{r}-9.9027 \\
(0.000)\end{array}$ & $\begin{array}{r}-12.1589 \\
(0.000)\end{array}$ & $\begin{array}{r}-6.2746 \\
(0.000)\end{array}$ & $\begin{array}{r}-8.3093 \\
(0.000)\end{array}$ & $\begin{array}{r}-13.8863 \\
(0.000)\end{array}$ \\
\hline Observações & 678 & 678 & 678 & 455 & 455 & 455 \\
\hline $\mathrm{R}^{2}$ & 0.67 & 0.57 & 0.56 & 0.72 & 0.62 & 0.60 \\
\hline VIF & $\begin{array}{r}2.65 \\
(0.378)\end{array}$ & - & - & $\begin{array}{r}2.41 \\
(0.414)\end{array}$ & - & - \\
\hline $\mathrm{RHO}$ & - & 0.335 & 0.671 & - & 0.4844 & 0.836 \\
\hline Hausman & - & $\begin{array}{r}49.12 \\
(0.000)\end{array}$ & - & - & $\begin{array}{r}74.70 \\
(0.000)\end{array}$ & - \\
\hline Breusch-Pagan Test & - & $\begin{array}{l}411.41 \\
(0.000)\end{array}$ & - & - & $\begin{array}{l}297.19 \\
(0.000)\end{array}$ & - \\
\hline
\end{tabular}

Notas: 1. Os valores entre parênteses representam o p-valor. 2. Nos modelos (III) e (VI) os coeficientes das variáveis (it) foram calculados pelo estimador de efeito fixo. 3. As variáveis expressas em valores monetários foram tomadas em log. 
Observa-se, inicialmente, o ganho de eficiência do modelo pelo uso de dados em painel. Note-se que no modelo de dados agrupados ESCOL e INFLAÇÃO são não significativas, especialmente a primeira. Diferentemente, em todas as outras regressóes onde a técnica de dados em painel é utilizada, essas variáveis mostram-se significantes.

Conforme já foi assinalado, o teste de Hausman indica a presença de correlação entre o componente individual e os regressores. No entanto, esta correlação não é verificada para todas as variáveis explicativas, pois nota-se diferença significativa entre os resultados gerados por efeito aleatório e fixo (Cornwell e Rupert, 1988) apenas para duas variáveis, ESCOL e ABERT. Preliminarmente, pode-se adiantar que se a heterogeneidade entre os países for levada em conta, a importância de se ter um ambiente favorável à entrada de investimentos externo, representada aqui pelo grau de abertura comercial, diminui, enquanto a qualificação da mão-de-obra tem sua influência elevada.

\section{VERIFICANDO A EXISTÊNCIA DE CAUSALIDADE ENTRE IDE E PIB}

Na seção anterior detectamos a existência de correlação contemporânea entre IDE e crescimento econômico, este último representado pela taxa média de crescimento dos últimos cinco anos. No entanto, não se pode afirmar categoricamente que é o crescimento do nível de atividade que causa os investimentos externos, apesar de esse ser um argumento bastante razoável, na medida em que o crescimento permite aos investidores externos prever lucros crescentes, por exemplo. Da mesma forma, é possível argumentar que o aumento de investimentos externos em países em desenvolvimento, ao trazer mais conhecimento e capital, constitui um importante coadjuvante para o processo de crescimento econômico. Como mostrado na seção 2, diversos trabalhos exploram esta hipótese.

Nair-Reicheit e Weinhold (2001) tentam mostrar a relação de causalidade entre crescimento e investimento utilizando um procedimento parecido com o que aparece em Holtz-Eakin et alii (1988) e que consiste em estimar, por variável instrumental, a seguinte relação:

$$
y_{i t}-y_{i t-1}=\sum_{j=1}^{m} \alpha_{j}\left(y_{i t-j}-y_{i t-j-1)}+\sum_{j=1}^{m} \delta_{j}\left(x_{i t-j}-x_{i t-j-1}\right)+\left(u_{i t}-u_{i t-1}\right)\right.
$$

A causalidade de $\mathrm{x}$ (IDE) no sentido de y (PIB) é aceita caso a hipótese conjunta $\delta_{1}=\delta_{2}=\ldots=\delta_{m}=0$ seja verdadeira. Aqui o uso da primeira diferença permite eliminar o efeito tixo. $O$ ponto fraco do trabalho de Nair-Reicheit e Weinhold (2001) consiste no fato de que para o teste de causalidade ser consistente é necessário mostrar que não existe causalidade reversa, tal como é feito na abordagem de Granger (1969) para 
séries de tempo. Assim, é necessário estimar um sistema de equações envolvendo as duas variáveis, e não apenas uma equação, como aparece em Nair-Reicheit e Weinhold (2001), ou seja, deve-se estimar um painel auto-regressivo (PVAR), tal como é mostrado a seguir. ${ }^{8}$

$$
\begin{aligned}
& I D E_{t}=\alpha+\sum_{i=1}^{m} \beta_{1 i} I D E_{t-i}+\sum_{i=1}^{m} \gamma_{1 i} P I B_{t-i}+\varepsilon_{t} \\
& P I B_{t}=\alpha+\sum_{i=1}^{m} \beta_{2 i} I D E_{t-i}+\sum_{j=1}^{m} \gamma_{2 i} P I B_{t-j}+v_{t}
\end{aligned}
$$

No teste de causalidade no sentido de Granger ${ }^{9}$ quatro situações são possíveis: a) IDE causa PIB, porém o contrário não é verdadeiro; b) PIB causa IDE, contudo o contrário não é fato; c) $\mathrm{IDE}$ causa $\mathrm{PIB}$, sendo o contrário também verdadeiro (bicausalidade); d) IDE não causa PIB, nem PIB causa IDE (sem relação de causalidade). Para aceitar a causalidade de Granger no sentido de que IDE causa PIB é necessário ter evidência que a hipótese (a) seja verdadeira.

No contexto de séries de tempo, em que o procedimento de Granger foi inicialmente aplicado, a estimação por OLS gera estimadores não viesados e consistentes para as equaçóes (5)-(6). No que concerne a dados de painel, o procedimento similar se daria a partir da aplicação do estimador LSDV para cada equação. No entanto, no que concerne a dados em painel isso está longe de ser verdadeiro. Neste caso, o procedimento usual de estimação do modelo por efeito fixo (LSDV) gera um estimador não consistente para um painel dinâmico. (Hsiao, 2003). Nickell (1981) obtém uma expressão para o viés de $\beta$, mostrando que o viés se aproxima de zero quando o número de observações temporais $\mathrm{T}$ tende para $\mathrm{o}$ infinito.

De modo a testar a causalidade entre IDE e PIB nesta pesquisa, faremos uso de duas abordagens na estrutura de dados em painel. O primeiro procedimento aparece originalmente em Carroll e Weil (1994) e Judson e Owen (1999), que fazem uso do estimador AH (Anderson e Hsiao, 1981), que utiliza dados macroeconômicos de painel para investigar a relação de longo prazo entre taxa de poupança e crescimento da renda per capita para diversos países. Anderson e Hsiao (1981) propóem eliminar o efeito fixo por meio do uso da primeira diferença e, em seguida, aplicar o método de variáveis instrumentais sobre a primeira diferença que, utilizando apenas a equação (5), é expressa da seguinte forma: ${ }^{10}$

8 Aqui novamente tomaremos ambas as variáveis em log.

9 Deve-se estar atento ao fato de que no teste de causalidade de Granger esta é vista no sentido de precedência temporal entre variáveis.

10 Tendo em vista a economia de espaço, utilizamos aqui apenas uma defasagem. Para um número maior de defasagens, o procedimento é idêntico. 


$$
I D E_{i t}-I D E_{i t-1}=\beta_{1}\left(I D E_{i t-1}-I D E_{i t-2}\right)+\delta_{1}\left(P I B_{i t-1}-P I B_{i t-2}\right)+\left(u_{1 i t}-u_{1 t-1}\right)(7)
$$

Observe-se que, em (7), o distúrbio $u_{1 i t}-u_{1 t-1}$ é agora correlacionado com a variável independente $L I D E_{i t-1}-L I D E_{i t-2}$. Anderson e Hsiao (1981) recomendam como instrumento $L I D E_{i t-2}-L I D E_{i t-3}$ ou $L I D E_{i t-2}$. Aqui a causalidade de Granger, no sentido de que $L P I B$ causa $L I D E$, é demonstrada caso a hipótese nula que $\delta_{1}=0$ não se confirme.

O segundo método a ser empregado neste estudo faz uso do estimador GMM de Arellano-Bond (1991). Este método propõe utilizar como instrumentos variáveis com defasagens de pelo menos dois períodos. Este estimador permite obter, para painéis dinâmicos, estimativas mais eficientes que as obtidas pelo estimador AH. Para testar qualquer relação de causalidade entre as variáveis que aparecem em (5)-(6) iremos estimar tais equações separadamente. Por hipótese, temos que $L P I B_{i t-1}$ é exógena em (5) e $L I D E_{i t-1}$ é exógena na equação (6). Conforme pode ser visto, ambas as equações incluem como regressor a variável dependente defasada.

A Tabela 2 apresenta os resultados da estimação das equações (5)-(6) decorrente da aplicação dos estimadores AH (Anderson e Hsiao, 1981) e AB (Arellano e Bond, 1991). A partir destes resultados é possível obter o sentido da causalidade de Granger entre IDE e PIB por meio da significância dos coeficientes estimados, tal como aparece na equação (4). A Tabela 2 também mostra os resultados obtidos por efeito fixo (LSDV) para o modelo dinâmico. A amostra usada envolve dados completos de 1975 a 2000. Devido à perda de grau de liberdade em decorrência do pequeno tamanho da amostra temporal, ${ }^{11}$ as equaçôes (5)-(6) foram estimadas somente até a terceira defasagem. Além disso, a introdução de muitas defasagens, embora diminua a autocorrelação serial, pode gerar problema de colinearidade entre as variáveis explicativas. Embora não seja mostrado, o modelo da Tabela 2 foi estimado também com duas defasagens. O modelo de uma defasagem seria pouco eficiente para eliminar a existência de autocorrelação serial.

Na Tabela 2 aparecem três testes de especificação para o modelo de Arellano-Bond. Primeiro, o teste de Sargan, que verifica a existência de sobreidentificação das restrições, tem como hipótese a validade conjunta dos instrumentos. Os demais testes $\operatorname{AR}(1)$ e $\operatorname{AR}(2)$, a existência de autocorrelação residual, respectivamente, de ordem um e dois. AR(1) é esperada na primeira diferença, enquanto a autocorrelação de ordens mais elevadas indica que algumas defasagens da variável dependente, que deve ser utilizada como instrumento, é na verdade endógena. Pode ser visto a partir das colunas (V)

11 Como os estimadores $\mathrm{AH}$ e $\mathrm{AB}$ são estimados por variáveis em diferença e não em nível, a perda de grau de liberdade é uma defasagem acima da última defasagem posta na regressão. 
e (VI) da Tabela 2 que os resultados destes testes não assinalam problemas de especificação.

TABELA 2 - CAUSALIDADE DE GRANGER PARA DADOS EM PAINEL (IDE X $P I B)$

\begin{tabular}{|c|c|c|c|c|c|c|}
\hline $\begin{array}{l}\text { Variáveis } \\
\text { Independentes }\end{array}$ & $\begin{array}{l}\text { D.IDE } \\
\text { LSDV } \\
\text { (I) }\end{array}$ & $\begin{array}{l}\text { D.PIB } \\
\text { LSDV } \\
\text { (II) }\end{array}$ & $\begin{array}{l}\text { D.IDE } \\
\text { AH } \\
\text { (III) }\end{array}$ & $\begin{array}{l}\text { D.PIB } \\
\text { AH } \\
\text { (IV) }\end{array}$ & $\begin{array}{l}\text { D.IDE } \\
\text { AB } \\
\text { (V) }\end{array}$ & $\begin{array}{l}\text { D.PIB } \\
\text { AB } \\
\text { (VI) }\end{array}$ \\
\hline L_1D.IDE & $\begin{array}{c}0.747 \\
(0,000)\end{array}$ & $\begin{array}{c}0.086 \\
(0.002)\end{array}$ & $\begin{array}{l}-0.246 \\
(0.000)\end{array}$ & $\begin{array}{c}0.023 \\
(0.002)\end{array}$ & $\begin{array}{c}0.483 \\
(0.000)\end{array}$ & $\begin{array}{c}0.021 \\
(0.008)\end{array}$ \\
\hline L_2D.IDE & $\begin{array}{c}0.044 \\
(0.449)\end{array}$ & $\begin{array}{l}-0.004 \\
(0.863)\end{array}$ & $\begin{array}{c}0.027 \\
(0.548)\end{array}$ & $\begin{array}{c}0.010 \\
(0.170)\end{array}$ & $\begin{array}{c}0.107 \\
(0.024)\end{array}$ & $\begin{array}{l}-0.011 \\
(0.158)\end{array}$ \\
\hline L_3D.IDE & $\begin{array}{l}-0.094 \\
(0.050)\end{array}$ & $\begin{array}{l}-0.010 \\
(0.564)\end{array}$ & $\begin{array}{l}-0.077 \\
(0.099)\end{array}$ & $\begin{array}{l}-0.002 \\
(0.720)\end{array}$ & $\begin{array}{c}0.005 \\
(0.890)\end{array}$ & $\begin{array}{l}-0.016 \\
(0.030)\end{array}$ \\
\hline L_1D.PIB & $\begin{array}{c}0.151 \\
(0.226)\end{array}$ & $\begin{array}{c}0.447 \\
(0.000)\end{array}$ & $\begin{array}{c}0.467 \\
(0.010)\end{array}$ & $\begin{array}{c}0.162 \\
(0,000)\end{array}$ & $\begin{array}{c}0.287 \\
(0.308)\end{array}$ & $\begin{array}{c}0.972 \\
(0.000)\end{array}$ \\
\hline L_2D.PIB & $\begin{array}{l}-0.160 \\
(0.260)\end{array}$ & $\begin{array}{l}-0.006 \\
(0.897)\end{array}$ & $\begin{array}{c}0.315 \\
(0.273)\end{array}$ & $\begin{array}{l}-0.059 \\
(0.197)\end{array}$ & $\begin{array}{l}-0.287 \\
(0.629)\end{array}$ & $\begin{array}{l}-0.244 \\
(0.000)\end{array}$ \\
\hline L_3D.PIB & $\begin{array}{c}0.022 \\
(0,837)\end{array}$ & $\begin{array}{l}-0.081 \\
(0.053)\end{array}$ & $\begin{array}{l}-1.166 \\
(0.000)\end{array}$ & $\begin{array}{c}-0.57 \\
(0.203)\end{array}$ & $\begin{array}{l}-0.907 \\
(0.001)\end{array}$ & $\begin{array}{c}0.011 \\
(0.750)\end{array}$ \\
\hline Constant & $\begin{array}{c}1.631 \\
(0.060)\end{array}$ & $\begin{array}{c}6.366 \\
(0.000)\end{array}$ & $\begin{array}{c}0.225 \\
(0.000)\end{array}$ & $\begin{array}{c}0.046 \\
(0.000)\end{array}$ & $\begin{array}{c}0.120 \\
(0.000)\end{array}$ & $\begin{array}{c}0.015 \\
(0.000)\end{array}$ \\
\hline Test AR(1) & - & - & - & - & $\begin{array}{l}302.73 \\
(0.350)\end{array}$ & $\begin{array}{l}-15.63 \\
(0.000)\end{array}$ \\
\hline Test AR(2) & - & - & - & - & $\begin{array}{l}-15.61 \\
(0.000)\end{array}$ & $\begin{array}{c}0.52 \\
(0.231)\end{array}$ \\
\hline $\begin{array}{c}\text { Sargan } \\
\text { Test }\end{array}$ & - & - & - & - & $\begin{array}{l}-1.150 \\
(0.231)\end{array}$ & $\begin{array}{l}310.55 \\
(0.270)\end{array}$ \\
\hline
\end{tabular}

Observaçôes: 1 . Todas as variáveis foram tomadas no log. 2. Os valores entre parênteses representam o P-valor. 3. $\mathrm{D} \cdot \operatorname{VAR}=\operatorname{VAR}(\mathrm{t})-\operatorname{VAR}(\mathrm{t}-\mathrm{l}), \quad \mathrm{L} \_1 \mathrm{D} \cdot \operatorname{IDE}=\operatorname{VAR}(\mathrm{t}-1)-\operatorname{VAR}(\mathrm{t}-2)$, L_2D.IDE $=\operatorname{VAR}(\mathrm{t}-2)-\operatorname{VAR}(\mathrm{t}-3)$.

De acordo com o que é visto na Tabela 2, os resultados acenam em direção à existência de causalidade simultânea entre IDE e PIB. Vejamos inicialmente o que ocorre na equação para IDE, colunas (III) e (V). No caso dos resultados obtidos a partir do emprego do estimador $\mathrm{AH}$, observa-se que o PIB mostra significância na primeira e terceira defasagens, enquanto que no que se refere ao resultado gerado por $A B$, a significância é percebida apenas na terceira defasagem. Em relação à equação do PIB (colunas IV e VI), tendo em vista os resultados gerados por AH, coluna (IV), verificase que o IDE apresenta significância apenas na primeira defasagem. Observando agora o que foi obtido pelo estimador AB, coluna (VI), podemos constatar que o IDE apre- 
senta significância para a primeira e terceira defasagens. Comparando os resultados obtidos por meio do uso desses dois estimadores para ambas as equações do IDE e PIB, observa-se uma certa assimetria nos resultados no que diz respeito à significância nas defasagens das variáveis.

Quanto aos resultados obtidos por LSDV, verifica-se a ocorrência inequívoca da causalidade no sentido de IDE afetar positivamente o PIB, e não o contrário. Sabe-se, entretanto, que o estimador LSDV é um estimador não consistente para painel dinâmico. Tomando por base o que acabamos de expor, somos levados a concluir que existe boa dose de evidência quanto à existência de causalidade simultânea entre IDE e PIB. Comparando os resultados gerados por LSDV com aqueles gerados pelo emprego dos estimadores de Arellano-Bond e Anderson-Hsiao, temos que esses últimos permitem identificar a causalidade reversa. Observa-se nas colunas (III) e (V) que a significância do PIB somente é alcançada na terceira defasagem, isto é, para a variável L_3.DPIB. Diferentemente, o impacto do IDE sobre o produto é verificado em menor espaço de tempo.

\section{CONCLUSÕES}

Basicamente, os determinantes dos investimentos diretos externos podem ser relativos às firmas e a características dos países de origem - push factors - ou a fatores locacionais - pull factors. A maior parte da literatura teórica relativa a empresas multinacionais nos últimos quarenta anos, justamente por focar sua atenção nas empresas, enfatizou os fatores relacionados a vantagens das firmas, dando pouca importância aos fatores macroeconômicos, tanto nos países de origem quanto nos países de destino dos investimentos.

Em parte, isso também se deveu ao fato de, por algum tempo, a pergunta relevante ter sido por que uma firma estrangeira podia concorrer em condições favoráveis com uma firma doméstica. Dito de outra forma, a questão que mais intrigava os pesquisadores era por que as empresas saíam de seus países para produzir no exterior ao invés de exportar. Talvez hoje, quando as grandes corporaçóes já estão instaladas num espaço global, a pergunta mais interessante seja por que elas escolhem alguns destinos e não outros.

Por outro lado, a maior parte dos estudos empíricos que examinam os determinantes dos investimentos diretos externos dá mais ênfase aos fatores locacionais. Um fator que contribui para explicar essa discrepância é o fato de ser extremamente difícil levantar dados relacionados a características das firmas para um grande número de países, especialmente em desenvolvimento. 
Foi analisada uma amostra de 38 países em desenvolvimento (inclusive economias em transição) para o período 1975-2000, com dados em painel, para estimar os determinantes dos fluxos de investimentos diretos externos. Ficou evidenciado, em primeiro lugar, que tanto o tamanho da economia, medido pelo PIB, quanto o ritmo de crescimento médio dos anos anteriores (G5PIB) afetam positivamente o ingresso de IDE e são fortemente significativos.

O grau de escolaridade (ESCOL) também aparece como um importante determinante de IDE e altamente significativo. Isto demonstra que boa parte dos investimentos diretos para países em desenvolvimento destina-se a atividades relativamente intensivas em conhecimento e que o aumento do grau de escolaridade pode ser uma política de indução desses investimentos.

O coeficiente de abertura da economia (ABERT), que foi incluído como proxy para a disposição do país aceitar investimentos estrangeiros, mostrou-se um fator importante para a atração de capital, tendo em vista que essa variável apresentou o sinal esperado, sendo altamente significativa.

A inflação (INFLAÇÃO) aparece como indicador da estabilidade macroeconômica, com sinal negativo e significativo na amostra maior. Entretanto, na amostra menor, com a introdução da variável RISCO, ela não é significativa nos padrões usuais, apesar de seu p-valor não ser excessivamente alto. No entanto, o risco do país mostrou importante efeito negativo sobre a entrada de investimentos diretos.

Uma das duas variáveis relativas apenas aos países de origem dos IDEs, o DOW JONES indicou que o crescimento dos mercados de capitais nos países desenvolvidos é um forte determinante dos fluxos de saída desses investimentos, sobretudo nos últimos anos.

Por fim, dado ainda que há muito debate a respeito da relação de causalidade entre investimentos diretos externos e produto, foi feito o teste de causalidade entre IDE e PIB. Nele não se constatou a existência inequívoca de causalidade num sentido específico, no qual o investimento direto externo sobre PIB não está assegurado. Diferentemente, o teste de causalidade no sentido de Granger apontou para a existência de causalidade simultânea entre IDE e PIB, isto é, os resultados parecem indicar que não existe um sentido específico, tal como se costuma colocar, em que o IDE afeta o PIB. Assim, por exemplo, o fato da China ser a maior economia em desenvolvimento do mundo, e a que apresenta uma das maiores taxas de crescimento nos últimos anos, certamente contribuiu para que se tornasse um dos maiores receptores de capital. Outro ponto interessante é que, de acordo com os resultados alcançados, o efeito dos IDEs 
sobre o PIB parece se mostrar mais presente no curto prazo, enquanto o efeito da causalidade reversa aparece num período de tempo mais distante.

APENNDICE

A) LISTA DOS PAÍSES HOSPEDEIROS:

$\begin{array}{lll}\text { África do Sul } & \text { Filipinas } & \text { Qatar } \\ \text { Angola } & \text { Índia } & \text { Rússia } \\ \text { Argentina } & \text { Indonésia } & \text { Senegal } \\ \text { Bolívia } & \text { Líbano } & \text { Tailândia } \\ \text { Brasil } & \text { Malásia } & \text { Turquia } \\ \text { Bulgária } & \text { Marrocos } & \text { Ucrânia } \\ \text { Chile } & \text { México } & \text { Uruguai } \\ \text { China } & \text { Moçambique } & \text { Venezuela } \\ \text { Cingapura } & \text { Nigéria } & \text { Vietnã } \\ \text { Colômbia } & \text { Panamá } & \text { Zâmbia } \\ \text { Coréia do Sul } & \text { Paquistão } & \\ \text { Costa do Marfim } & \text { Paraguai } & \\ \text { Egito } & \text { Peru } & \\ \text { Equador } & \text { Polônia } & \end{array}$

\section{B) LISTA DOS PAÍSES SELECIONADOS DA OECD:}

Alemanha
Bélgica
Canadá
Espanha
Estados Unidos

França

Holanda

Japão

Luxemburgo

Suíça 
C) FONTES DOS DADOS:

IDE e PIB - International Financial Statistics do FMI.

INFL, ESCOL, ENERCON, ABERT e OECDGROWTH - World Development Indicators do Banco Mundial.

DOWJONES - IPEADATA.

RISCO - Euromoney.

\section{BIBLIOGRAFIA}

Anderson, T. W.; Hsiao, C. Estimation of dynamic models with error components. Journal of the American Statistical Association, 76, p. 589-606, 1981.

Arellano, M.; Bond, S. Some tests of specification for panel data: Monte Carlo evidence and an application to employent equations. The Review of Economic Studies 58, p. 277-297, 1991.

Baltagi, B. H. Econometric analysis of panel data. John Wiley e Sons, 1995.

Borenstein, Eduardo; Gregorio, José de; Lee, J. W. How does foreign direct investment affect economic growth? Working Paper n. 5057, Cambridge, MA: NBER, 1995.

Buckley, Peter; Casson, Mark. The future of the multinational enterprise. London: Mac Millan, 1976.

. The optimal timing of a foreign direct investment. Economic Journal, v. 91, March 1981.

Buckley, Peter J.; Ghauri, Pervez N. The internationalization of the firm: a reader. 1991.

Buckley, Peter J.; Clegg, Jeremy; Wang, Chengpi; Cross, Adam R. FDI, regional differences and economic groth: panel data evidence from China. Transnational Corporations, v. 11, n. 1, Abril 2002.

Campos, Nauro F.; Kinoshita, Yuko. Why does FDI go where it goes? New evidence from the transition economies. IMF Working Paper, IMF Institute, Novembro de 2003 .

Cantwell, John. A survey of theories of international production. In: Pitelis, C.; Sugden, R., The nature of the transnational firm. London: Routledge, $2000(a)$.

. Notas manuscritas apresentadas no Workshop on Mergers and Acquisitions and Globalization of Japanese Firms. Instituto de Economia/UFRJ, outubro de 2000 (b). 
Carrol, C.; Weil, D. Saving and growth: a reinterpretation. Carnegie-Rochester Conference Series on Public Policy 40, p. 133-192, 1994.

Caves, R. E. International corporations: the industrial economics of foreign investment. Economica, v. 38, February 1971.

Cornwell, C.; Rupert, P. Efficient estimation with panel data: an empirical comparison of instrumental variables. Joumal of Applied Econometrics, 3, p. 149-155, 1988.

Dunning, J. Multinational enterprise and the global economy. Wokinghan: AddisonWesley, 1993.

Determinants of foreign direct investment: globalization induced changes and the role of FDI policies. Annual Bank Conference on Development Economics, 2002.

Garibaldi, Pietro; Mora, Nada; Sahay, Ratna; Zettelmeyer, Jeromin. What moves capital to transition economies. IMF Staff Papers, v. 48, Special Issue, International Monetary Found, 2001.

Graham, Edward. Strategic management and transnational firm behaviour: a formal approach. In: Pitelis, C.; Sugden, R. The nature of the transnational firm. $2^{\text {nd }}$ edition. London: Routledge, 2000.

. Market structure and the multinational enterprise: a game theoretic approach. Journal of International Business Studies, v. 29, n. 1, 1998.

. Transatlantic investment by multinational firms: A rivalistic phenomenon? Journal of Post-Keynesian Economics, v. 1, n. 1, Fall 1978.

Granger, C. Investigating causal relations by econometric model and cross-spectral methods. Econometrica, 37, p. 424-438, 1969.

Greene, W. Econometric analysis. Prentice Hall, 1993.

Hausman, J. A.; Taylor, W. Panel data and unobservable individual effects. Econometrica, v. 49, n. 6, p. 1377-1398, 1981.

Holtz-Eakin, D.; Newey, W.; Rosen, H. Estimating vector autoregressions with panel data. Econometrica, v. 56, n. 6, p. 1371-1395, 1988.

Holland, Dawn; Sass, Magdolna; Benacek, Vladimir; Gronicki, Miroslaw. The determinants and impact of FDI in central and eastern Europe: a comparison of survey and econometric evidence. Transnacional Corporations, v. 9, n. 3, Dezembro 2000.

Hsiao, C. Analysis of panel data. Second edition. Cambridge University Press, 2003.

Hymer, S. The international operations of national firms: a study of direct foreign investment. 1976. PhD Dissertation, MIT, Publicada por MIT Press.

Judge, G.; Hill, C.; Griffiths, W.; Lee, T.; Lütkepohl, H. Introduction to the theory and practice of econometrics. New York: Wiley, 1982. 
Judson, R. A.; Owen, A. L. Estimating dynamic panel data models: a guide for macroeconomists. Economic Letters, 65, p. 9-15, 1999.

Kindlegerger, C. P. American business abroad: six lectures on direct investment. New Heaven: Yale University Press, 1969.

Lipsey, Robert E. Inward FDI and economic growth in developing countries. Transnational Corporations, v. 9, n. 1, Abril 2000.

Loree, David W.; Guisinger, Stephen E. Policy and non-policy determinants os U. S. equity foreign direct investment. Journal of International Business Studies, v. 26, issue 2, p. 281-299, 1995.

Mello, Luiz R. de. Foreign direct investment-led growth: evidence from time series and panel data. Oxford Economic Papers 51, p. 199-151, 1999.

Markusen,-James-R; Venables, Anthony-J. Multinational firms and the new trade theory. National Bureau of Economic Research Working Paper, Fevereiro 1995.

Nair-Reicheit, U.; Weinhold, D. Causality tests for cross-country panels: new look at FDI and economic growth in developing countries. Oxford Bulletin of Economics and Statistics, v. 63, n. 2, p. 151-171, 2001.

Nickell, S. Biases in dynamic models with fixed effects. Econometrica, 49, p. 1399$1416,1981$.

Nonnenberg, Marcelo José B. Determinantes dos investimentos externos e impactos das empresas multinacionais no Brasil - 1956/2000. 2002. Tese (Doutorado), apresentada ao Instituto de Economia, UFRJ.

Nunnenkamp, Peter; Spatz, Julius. Determinants of FDI in developing countries: has globalization changed the rules of the game? Transnacional Corporations, v. 11, n. 2, Agosto 2002.

Ohlin, Bertil. [1933]. Comercio interregional e internacional. Barcelona: Oikos, 1971.

Soto, Marcelo. Capital flows and growth in developing countries: recent empirical evidence. OECD Development Centre. Technical Papers 160, Julho de 2000.

Tsai, Pan-Long. Determinants of foreign direct investment and its impact on economic growth. Journal of Economic Development, v. 19, n. 1, Junho 1994.

Vernon, R. International investment and international trade in the product cycle. Quarterly Journal of Economics, v. 80, 1966.

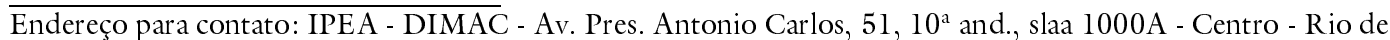
Janeiro - RJ - CEP 20020-010.

E-mails: mjorge@ipea.gov.br; nonnemberg@ipea.gov.br.

(Recebido em abril de 2004. Aceito para publicação em junho de 2005). 